\title{
Chromoplexy: a new paradigm in genome remodeling and evolution
}

\author{
Kendric Wang ${ }^{1}$, Yuzhuo Wang ${ }^{1,2}$ and Colin C Collins ${ }^{1}$ \\ Asian Journal of Andrology (2013) 15, 711-712; doi:10.1038/aja.2013.109; Published online: 26 August 2013
}

E arly massively-parallel sequencing studies have revealed the mutational landscape of protein-coding genes in prostate cancer. However, most of these studies have not explored the extensive influence of genomic rearrangement in prostate cancer. In a recent Cell article, Baca and colleagues used whole-genome sequencing to tackle this issue, comprehensively surveying the abundance of genomic rearrangements present in a large cohort of 57 prostate cancers. They characterized a wide-spread phenomenon termed 'chromoplexy', which may drive cancer evolution through the phenomena of punctuated equilibrium by concurrently dysregulating numerous cancer genes across multiple chromosomes. While the causes of this event still require elucidation, this defining discovery undoubtedly offers an important glimpse into the evolutionary process of prostate cancer.

Genomic rearrangements are highly prevalent in prostate cancer, have a profound impact on tumor development and progression (reviewed in Ref. ${ }^{1}$ ), and may serve as promising prognostic biomarkers. ${ }^{2,3}$ For example, numerous recurrently gained or lost regions are linked to poor clinical outcome, such as MYC amplifications at $8 \mathrm{q} 24$ and PTEN deletions at $10 \mathrm{q} 23 .{ }^{4}$ Also, gene fusions involving ETS-family transcription factors (e.g., TMPRSS2-ERG) are considered an early carcinogenic event and are present in up to $70 \%$ of Western prostate cancers. ${ }^{5}$ Whereas microarray-based studies were largely limited to the analysis of copy number aberrations, in prostate cancer, massivelyparallel sequencing-based technologies allow

${ }^{1}$ Vancouver Prostate Centre \& Department of Urologic Sciences, University of British Columbia, Vancouver, BC V6H 3Z6, Canada and ${ }^{2}$ Department of Experimental Therapeutics, BC Cancer Agency, Vancouver, BC V5Z 1L3, Canada

Correspondence: Dr CC Collins (ccollins@

prostatecentre.com) investigation of far more complex structural variants.

The first whole-genome studies reported complex genomic rearrangements involving multiple genes. ${ }^{6-8}$ These complex rearrangements can produce 'poly-gene fusions' disrupting multiple genes simultaneously, or form 'closed-chains of breakage and rejoining', which are analogous to the breaking, shuffling and rejoining of many regions in a closed chain rearrangement. The involvement of the TMPRSS2-ERG gene fusion in several of these complex events suggested their relevance to prostate cancer initiation, ${ }^{6}$ and laid the foundation for the significant followup study carried out by Baca et al. Surprisingly, they revealed these complex genomic events to be a wide-spread phenomenon in prostate cancer and termed the phenomenon 'chromoplexy' to reflect the complex weaving or restructuring of the genome. ${ }^{9}$

Baca et al. expanded on prior observations of chained rearrangements ${ }^{6}$ and developed a computational method to systematically detect chromoplexy events. Surprisingly, almost $90 \%$ of the 57 malignant tumors analyzed contained a chain consisting of five or more rearrangements and over $60 \%$ of tumors contained two or more of these chains. These chromoplexy events collectively involved almost $40 \%$ of total genomic rearrangements, suggesting a fundamental link to the etiology of genomic rearrangements in prostate cancer. Moreover, additional computational simulations revealed that these complex events most likely arise through a single, coordinated process, rather than independently through multiple, sequential steps.

Interestingly, Baca et al. observed a difference in the chromoplexy distribution across different molecular subtypes of prostate cancer defined by ETS fusion status and CHD1 mutation status. Tumors with the oncogenic ETS fusions $\left(\mathrm{ETS}^{+} / \mathrm{CHD}^{\mathrm{wt}}\right)$ harbored greater number of interchromosomal rearrangements, involving up to seven chromosomes in a single chain. A majority of ETS $^{+}$tumors had chromoplexy events that involved ERG fusions, which suggests chromoplexy may arise from the same transcriptional process driven by the androgen receptor (AR) that yields TMPRSS2-ERG fusions. ${ }^{10}$ This possibility was additionally supported by the enrichment of breakpoints from these chains in highly expressed genomic regions.

In contrast, the CHD1-deleted subset of $\mathrm{ETS}^{-}$tumors $\left(\mathrm{ETS}^{-} / \mathrm{CHD}^{\mathrm{del}}\right)$ featured more intrachromosomal rearrangements. In these tumors, where chained rearrangements were typically localized across only one or two chromosomes, there were more rearrangements overall (up to seven times the average number of rearrangements). The phenotypic differences between these two tumor subtypes may be related to the role of CHD1 as a chromatin-modifying tumor suppressor whose inactivation can suppress AR-transcriptional activity and prevent $E R G$-fusion formation. ${ }^{11}$ The enrichment of breakpoints in late-replicating DNA and heterochromatin regions with lower gene expression also supports an alternative cause of chromoplexy in ETS $^{-}$ tumors. Recently, SPOP has been implicated as a tumor suppressor gene recurrently mutated in $13 \%$ of prostate cancers and in mutually exclusivity to ETS family rearrangements. ${ }^{12}$ Since SPOP mutations and CHD1 deletions collectively define a large fraction of ETS ${ }^{-}$tumors, it remains to be seen if they can cooperate in shifting chromoplexy formation away from an AR-driven transcriptional mechanism.

The characteristics of chromoplexy are reminiscent of chromothripsis, another emerging phenomenon in cancer evolution. Whereas the classical view of cancer progression follows the gradual accumulation of 
mutations that promote cell survival and invasion, ${ }^{13}$ both chromoplexy and chromothripsis are large-scale genomic rearrangement events that can disrupt numerous essential cell processes in a single step. As such, these catastrophic events are predicted to be retained only by inducing oncogenic alterations that compensate the cell with strong selective advantages.

At the same time, chromoplexy differs from chromothripsis in a number of fundamental aspects. Chromoplexy affects fewer rearranged regions, numbering in the tens rather than hundreds, scattered across many chromosomes rather than localized in one or two chromosomes. ${ }^{14}$ Furthermore, chromoplexy appears to be a frequent phenomenon in prostate cancer, whereas the frequency of chromothripsis ranges between $2 \%$ and $3 \%$ in different cancers. ${ }^{14}$ Although the mechanisms of both phenomenon still need to be clearly understood, chromoplexy in prostate cancer may be caused by a number of processes including a transcriptional-related mechanism in $\mathrm{ETS}^{+}$tumors, whereas converging evidences suggest genome instability and micronuclei formation as a leading cause of chromothripsis. ${ }^{15}$

In the wake of this study, we eagerly anticipate follow-up to a number of important questions. Although features of chromoplexy in $\mathrm{ETS}^{+}$prostate tumors strongly suggests DNA damage induced by AR-driven transcription as a probable mechanism, observations of these complex genomic rearrangements in an androgen-stimulated in vitro model will be necessary to validate this hypothesis. Similarly, examining chromoplexy in other cancer systems removed from AR influence (e.g., breast cancer, small-cell lung cancer) may facilitate an understanding for this phenomenon in ETS $^{-}$tumors. Interestingly, like genome instability generally, the occurrence of chromoplexy appears to support a punctuated equilibrium model of evolution that proposes long periods of relative stability punctuated by sudden, rapid periods of radical change. Since the progression of prostate cancer may largely depend on multiple rounds of chromoplexy, elucidating the triggers for these periods of punctuated change can hold the key to locking prostate cancer in evolutionary stasis. Moreover, confirmation of punctuated equilibrium as a mechanism for cancer evolution will ultimately have profound implications for evolutionary biology as a whole.

1 Wyatt AW, Mo F, Wang Y, Collins CC. The diverse heterogeneity of molecular alterations in prostate cancer identified through next-generation sequencing. Asian J Androl 2013; 15: 301-8.

2 Paris PL, Weinberg V, Albo G, Roy R, Burke C et al. A group of genome-based biomarkers that add to a Kattan nomogram for predicting progression in men with high-risk prostate cancer. Clin Cancer Res 2010; 16: 195-202.
3 Taylor BS, Schultz N, Hieronymus H, Gopalan A, Xiao $\mathrm{Y}$ et al. Integrative genomic profiling of human prostate cancer. Cancer Cell 2010; 18: 11-22.

4 Kim JH, Dhanasekaran SM, Mehra R, Tomlins S, Gu W et al. Integrative analysis of genomic aberrations associated with prostate cancer progression. Cancer Res 2007; 67: 8229-39.

5 Tomlins SA, Laxman B, Dhanasekaran SM, Helgeson $\mathrm{BE}, \mathrm{Cao} \mathrm{X}$ et al. Distinct classes of chromosomal rearrangements create oncogenic ETS gene fusions in prostate cancer. Nature 2007; 448: 595-9.

6 Berger MF, Lawrence MS, Demichelis F, Drier Y, Cibulskis $\mathrm{K}$ et al. The genomic complexity of primary human prostate cancer. Nature 2011; 470: 214-20.

7 Wu C, Wyatt AW, Mcpherson A, Lin D, Mcconeghy BJ et al. Poly-gene fusion transcripts and chromothripsis in prostate cancer. Genes Chromosomes Cancer 2012; 1153: 1144-1153.

8 McPherson AW, Wu C, Wyatt A, Shah SP, Collins C et al. nFuse: discovery of complex genomic rearrangements in cancer using high-throughput sequencing. Genome Res 2012; 22: 2250-2261.

9 Baca SC, Prandi D, Lawrence MS, Mosquera JM, Romanel $\mathrm{A}$ et al. Punctuated evolution of prostate cancer genomes. Cell 2013; 153: 666-677.

10 Haffner MC, Aryee MJ, Toubaji A, Esopi DM, Albadine R et al. Androgen-induced TOP2B-mediated double-strand breaks and prostate cancer gene rearrangements. Nat Genet 2010; 42: 668-75.

11 Burkhardt L, Fuchs S, Krohn A, Masser S, Mader M et al. CHD1 is a $5 q 21$ tumor suppressor required for ERG rearrangement in prostate cancer. Cancer Res 2013; 73: 2795-805.

12 Barbieri CE, Baca SC, Lawrence MS, Demichelis F Blattner $\mathrm{M}$ et al. Exome sequencing identifies recurrent SPOP, FOXA1 and MED12 mutations in prostate cancer. Nat Genet 2012; 44: 685-9.

13 Stratton MR, Campbell PJ, Futreal PA. The cancer genome. Nature 2009; 458: 719-24.

14 Stephens PJ, Greenman CD, Fu B, Yang F, Bignell GR et al. Massive genomic rearrangement acquired in a single catastrophic event during cancer development. Cell 2011; 144: 27-40.

15 Crasta K, Ganem NJ, Dagher R, Lantermann AB, Ivanova EV et al. DNA breaks and chromosome pulverization from errors in mitosis. Nature 2012; 482: 53-8. 